Bull. Mater. Sci., Vol. 14, No. 4, August 1991, pp. 1079-1081. (C) Printed in India.

\title{
Role of apex oxygen and redox elements in high temperature superconductors
}

\author{
R E AMRITKAR, D G KANHERE, P V PANAT and \\ A P B SINHA
}

Department of Physics, University of Poona, Pune 411007 , India

\begin{abstract}
The presence of valence fuctuating redox ions such as $\mathrm{Pb}, \mathrm{T}, \mathrm{Sb}$, etc can enhance the transition temperature of the new oxide superconductors. We propose the possibility of the existence of negative Hubbard parameter $(U)$ on redox ion which results in effective negative $U$ on apex oxygen bridging redox ion and the $\mathrm{CuO}_{2}$ planes. This enhances $T_{c}$.
\end{abstract}

Keywords. Redox ions; apex oxygen.

\section{Model}

The discovery of high temperature superconductivity in oxide system of $\mathrm{La}$ or $\mathrm{Y}$ and many others have sparked immense interest in the physics community (Bednorz and Müller 1986; Wu et al 1987).

It is well known that $T_{c}$ increases roughly proportional to the number of $\mathrm{CuO}_{2}$ planes per block, e.g. $T_{c}$ of the oxide materials with triple perovskite structure is larger than $\mathrm{La}_{2} \mathrm{CuO}_{4}$ type structures. Most of the superconductors with $T_{c}>40 \mathrm{~K}$ can be arranged into an assembly of layers with the following sequence (Sinha 1989)

$$
R-B-S-(I-S)_{n-1}-B-R
$$

where $S$ is the superconducting $\mathrm{CuO}_{2}$ plane, $I$ an insulating layer such as calcium $\square / Y \square$ separating two adjacent $S$ planes, $R$ is a layer containing redox ions such as bismuth, thalium or lead etc. and $B$ is a bridge layer of oxygens which bridges $R$ and $S$ layer, $n$ is the number of $S$ layers per block and for $n=1$ the redox layer is absent. We also observe that for the same number $\mathrm{CuO}_{2}$ layers, $T_{c}$ is higher for those compounds which have a redox layer than those without it (e.g. $T_{c}$ of $\mathrm{La}_{2} \mathrm{CuO}_{4}$ is small as compared to that of $\mathrm{Tl}_{2} \mathrm{Ba}_{2} \mathrm{CuO}_{6}$ ). Chemically the redox ions have two stable valence states (e.g. $\mathrm{Bi}^{3+}$ and $\mathrm{Bi}^{5+}$ or $\mathrm{Tl}^{+}$and $\mathrm{Tl}^{3+}$ or $\mathrm{Pb}^{2+}$ and $\mathrm{Pb}^{4+}$ ). Oxygen from the bridge layer is directly coupled to the redox ion because of the apex position of it.

Two standard states of oxygen are $\mathrm{O}^{--}$and $\mathrm{O}^{\circ}$. This makes charge state $\left(\mathrm{Tl}^{3+}\right.$, $\left.\mathrm{Tl}^{+}\right)$with $\left(\mathrm{O}^{--}, \mathrm{O}^{0}\right)$ to have a lower energy than $\left(\mathrm{Tl}^{++}\right)$with $\left(\mathrm{O}^{-}\right)$. This is modelled by taking a negative Hubband parameter $U$ on redox ions.

Recently Khomskii and Zvezdin (1988) introduced negative $U$ on $\mathrm{CuO}_{2}$ plane in the hamiltonian to explain the high $T_{c}$ behaviour of the oxide superconductors. Varma (1988) introduced negative $U$ to explain the missing valence states of redox ions.

In view of the above discussion, we think it plausible that negative $U$ is placed on redox ion $\left(-U_{r}\right)$. This induces negative $U$ on apex oxygen $\left(-U_{p}\right)$ if we assume hybridization between them. For, consider a following model hamiltonian between an $R$ layer and the $B$ layer.

$$
H=\sum_{i, \sigma} \varepsilon_{b} b_{i \sigma}^{\dagger} b_{i \sigma}-U_{r} \sum_{i} b_{i \uparrow}^{\dagger} b_{i \uparrow} b_{i \downarrow}^{\dagger} b_{i \downarrow}+\sum_{i, \sigma} \varepsilon_{p} p_{i \sigma}^{\dagger} p_{i \sigma}+\sum_{i, \sigma}\left(V b_{i \sigma}^{\dagger} p_{i \sigma}+\text { h.c. }\right) .
$$


Here, $b_{i \sigma}$ and $p_{i \sigma}$ are annihilation operators for redox and oxygen sites having the same $z$ coordinates and index $i$ refers to $x-y$ coordinates in the plane. The last term gives the hybridization.

After making Schrieffer-Wolf transformations we get the effective hamiltonian as

$$
\begin{aligned}
H_{\mathrm{eff}}= & \sum_{i, \sigma}\left(\varepsilon_{b} b_{i \sigma}^{\dagger} b_{i \sigma}+\varepsilon_{p} p_{i \sigma}^{\dagger} p_{i \sigma}\right)-U_{r} \sum n_{i \uparrow}^{b} n_{i \downarrow}^{b} \\
& +B \sum_{i, \sigma}\left[n_{i \sigma}^{b} n_{i-\sigma}^{b}\left(1-n_{i \sigma}^{p}-n_{i-\sigma}^{p}\right)-n_{i \sigma}^{p} n_{i-\sigma}^{p}\left(1-n_{i \sigma}^{b}-n_{i-\sigma}^{b}\right)\right] .
\end{aligned}
$$

where $n_{i \sigma}^{b}$ is the number operator for redox ion on site $i$ and $n_{i \sigma}^{p}$ is correspondingly for apex oxygen at site $i$, and

$$
B=-2\left[\frac{1}{\varepsilon_{p}-\varepsilon_{b}}-\frac{1}{\varepsilon_{p}-\varepsilon_{b}-U_{r}}\right]^{2} \frac{|V|^{4}}{\left(2 \varepsilon_{b}-2 \varepsilon_{p}-U_{r}\right)} .
$$

Note $\varepsilon_{b} \sim \varepsilon_{p}$ and $B>0$. If we denote the basis states by $\left|n_{i \sigma}^{p}, n_{i-\sigma}^{p} ; n_{i \sigma}^{b}, n_{i-\sigma}^{b}\right\rangle$ then it is clear from (2) that, states $|0,0 ; 1,1\rangle$ with energy $2 \varepsilon_{b}-U_{r}+B$ and states $|1,1 ; 0,0\rangle$ with energy $2 \varepsilon_{p}-B$ are of lower energy than states such as $|1,0 ; 1,0\rangle$ with energy $\varepsilon_{b}+\varepsilon_{p}$. Thus we prove that there is effective negative $U$ on the apex oxygen.

To investigate the effect of negative Hubbard parameter $\left(-U_{P}\right)$ on $\mathrm{CuO}_{2}$ plane, consider the hamiltonian

$$
H=\sum_{k, \sigma} \varepsilon_{k} a_{k \sigma}^{\dagger} a_{k \sigma}+\sum_{i, \sigma} \varepsilon_{p} p_{i \sigma}^{\dagger} p_{i \sigma}-U_{P} \sum n_{i \uparrow}^{p} n_{i \downarrow}^{p}+\sum_{i, k, \sigma}\left(\tilde{V} a_{k \sigma}^{\dagger} p_{i \sigma}+\text { h.c. }\right) .
$$

Here, the first term represents conduction electron band in $\mathrm{CuO}_{2}$ layer with $a_{k \sigma}$ as annihilation operator of electron with momentum $k$ and spin $\sigma . \widetilde{V}$ is the hybridization term between conduction electron and an apex electron.

We show that this enhances attraction in the $\mathrm{CuO}_{2}$ plane. Following the Schrieffer-Wolf procedure, and assuming that $\varepsilon_{k} \sim \varepsilon_{F}$ we get the following effective hamiltonian,

$$
H_{\mathrm{eff}}=\sum_{k, \sigma} \varepsilon_{k} a_{k \sigma}^{\dagger} a_{k \sigma}-\sum_{k, k^{\prime}, \sigma} \frac{X^{2}}{\left(2 \varepsilon_{F}-2 \varepsilon_{g^{\prime}}+U_{P}\right)}\left[a_{k \sigma}^{\dagger} a_{k^{\prime}-\sigma}^{\dagger} a_{k^{\prime} \sigma} a_{k-\sigma}+\text { h.c. }\right] .
$$

where

$$
X=\tilde{V}^{2}\left[\frac{1}{\varepsilon_{F}-\varepsilon_{p}}-\frac{1}{\varepsilon_{F}-\varepsilon_{p}+U_{P}}\right]
$$

The interaction term in (4) depends upon energy denominators. Since $\varepsilon_{F}>\varepsilon_{p}, X^{2} /\left(2 \varepsilon_{F}-2 \varepsilon_{P}+U_{P}\right)>0$ resulting in enhanced effective attractive interaction between electron in the $\mathrm{CuO}_{2}$ planes. This attractive interaction is largest when $\varepsilon_{F} \sim \varepsilon_{P}$. We note that this attraction is obtained because of the virtual exchange of electrons in the $\mathrm{CuO}_{2}$ plane and the apex oxygen planes.

We do not expect this to be the main mechanism of superconductivity. However it will enhance the attractive interaction which may already be existing in the $\mathrm{CuO}_{2}$ planes due to other mechanisms. We see then that this mechanism couples different $\mathrm{CuO}_{2}$ planes giving a three-dimensional character to the superconductivity (Anderson 1989). 


\section{References}

Anderson P W 1989 Unpublished

Bednorz J G and Müller K A 1986 Z. Phys. B64 189

Khomskii D I and Zvezdin A K 1988 Solid State Commun. 66651

Sinha A P B 1989 in Studies of high temperature superconductors, (ed.) A V Narlikar (Nova Science Publisher Inc.)

Varma C M 1988 Phys. Rev. Lett. 612713

Wu M K et al 1987 Phys. Rev. Lett. 581891 